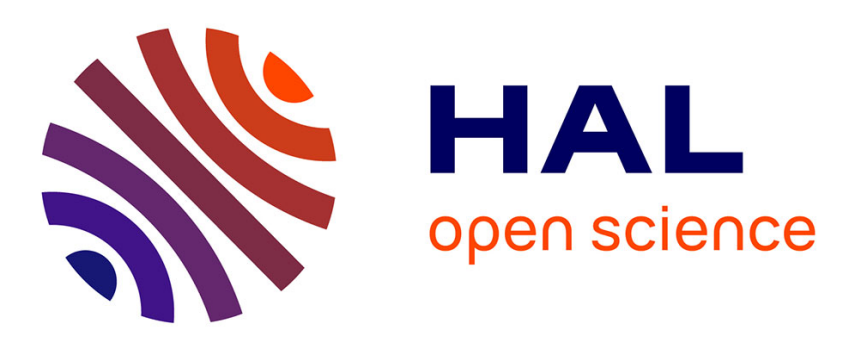

\title{
Parareal multi-model numerical zoom for parabolic multiscale problems
}

\author{
Franz Chouly, Alexei Lozinski
}

\section{To cite this version:}

Franz Chouly, Alexei Lozinski. Parareal multi-model numerical zoom for parabolic multiscale problems. Comptes Rendus. Mathématique, 2014, 352 (6), pp.535-540. hal-00934390

\author{
HAL Id: hal-00934390 \\ https://hal.science/hal-00934390
}

Submitted on 21 Jan 2014

HAL is a multi-disciplinary open access archive for the deposit and dissemination of scientific research documents, whether they are published or not. The documents may come from teaching and research institutions in France or abroad, or from public or private research centers.
L'archive ouverte pluridisciplinaire HAL, est destinée au dépôt et à la diffusion de documents scientifiques de niveau recherche, publiés ou non, émanant des établissements d'enseignement et de recherche français ou étrangers, des laboratoires publics ou privés. 
Numerical Analysis

\title{
Parareal multi-model numerical zoom for parabolic multiscale problems
}

\author{
Franz Chouly ${ }^{\mathrm{a}}$, Alexei Lozinski ${ }^{\mathrm{a}}$, \\ ${ }^{a}$ Laboratoire de Mathématiques de Besançon - UMR CNRS 6623, Université de Franche Comté, 16 route de Gray, 25030 \\ Besançon Cedex, France. \\ Received $* * * * *$; accepted after revision +++++ \\ Presented by $£ £ £ £ £$
}

\begin{abstract}
We present a time-parallel numerical zoom method for parabolic multiscale problems. A fine finite element solver is defined on a patch where multiscale effects are localized. A coarse finite element solver, based on a coarser timespace discretization, and eventually smoother than the fine one, is defined on the whole domain of the partial differential equation. The coupling between fine and coarse solvers is carried out with an adaptation of the Parareal algorithm. We detail our numerical method and illustrate it with a numerical experiment. To cite this article: F. Chouly, A. Lozinski, C. R. Acad. Sci. Paris, Ser. I 340 ().

\section{Résumé}

Une méthode de zoom numérique "pararéelle". Nous présentons une méthode de zoom numérique parallèle en temps pour les problèmes paraboliques multi-échelles. Sur un patch d'éléments finis qui contient les détails multi-échelles, nous définissons un solveur fin. Un solveur éléments finis grossier, basé sur une discrétisation spatiale et temporelle plus grossière, et éventuellement plus lisse que le fin, est défini sur le domaine complet de l'équation aux dérivées partielles. L'algorithme Parareal est adapté pour coupler ces solveurs fin et grossier. Nous décrivons dans le détail notre méthode, et nous l'illustrons via une expérience numérique. Pour citer cet article: F. Chouly, A. Lozinski, C. R. Acad. Sci. Paris, Ser. I 340 ().
\end{abstract}

\section{Introduction}

For multiscale problems where the multiscale effects are localized, a great variety of numerical methods have been proposed that are based on iterations between two grids: a coarse one on the whole domain, and a fine one on a patch which covers the region endowed with multiscale characteristics. To quote a few examples, let us mention the Chimera method [12], the method of finite element patches [6] or the numerical zoom [1]. Recently a new method has been proposed to couple different models on the

Email addresses: franz.chouly@univ-fcomte.fr (Franz Chouly), alexei.lozinski@univ-fcomte.fr (Alexei Lozinski). 
global domain and on the patch, where for instance the coarser model can be a smoothed version of the original one $[11,8]$. We show in this note that an extension for parabolic time-dependent problems of this multi-model numerical zoom can be derived using the Parareal framework [10].

The Parareal algorithm allows time-parallel time-integration of evolution equations $[10,3,4]$. This is of interest for long-time simulations of systems with a few degrees of freedom, or where the number of processors is so large that standard (spatial) domain decomposition techniques reach their limit because of bandwidth effects [4]. The stability and convergence properties of this algorithm have been studied for parabolic problems in, e.g., [5]. For the multi-model numerical zoom described in [11,8], a parareal time-integration presents attractive features since it allows a time-step for solution of the fine problem on the patch that can be finer than the time-step of the coarser problem on the global domain. Moreover, the finer computations, that require some extra cost, can then be parallelized to some extent. Note there exists recent works where the Parareal framework is adapted to multiscale problems, in the ODE context [9] and in the lattice-Boltzmann context [2].

We first describe the space semi-discretization for a simple parabolic multiscale problem, and then detail our Parareal time-integration method. We illustrate finally the potential interest of this new method with a numerical experiment.

\section{The multiscale problem and its space semi-discretization}

Let $\Omega \subset \mathbb{R}^{d}$ be an open bounded polygonal domain $(d \geq 1)$ and note $\partial \Omega$ its boundary. We consider the following diffusion problem:

$$
\frac{\partial u}{\partial t}-\nabla \cdot(\mathbf{K} \nabla u)=f \text { in } \Omega \times(0, T),\left.\quad u\right|_{\partial \Omega \times(0, T)}=0, \quad u(\cdot, t=0)=u_{0},
$$

where $u_{0}$ is the initial condition, $f$ is the source term, and where the diffusion matrix $\mathbf{K}=\left(K_{i j}\right)$, $1 \leq i, j \leq d$, satisfies $\alpha|\xi|^{2} \leq \mathbf{K}(\mathbf{x}, t) \xi \cdot \xi \leq \beta|\xi|^{2}$ for all $\xi \in \mathbb{R}^{d}$ and $(\mathbf{x}, t) \in \Omega \times(0, T)$ with some constants $0<\alpha \leq \beta<\infty$. We are interested here in the situation where the coefficients $K_{i j}$ are highly oscillating in a small subdomain $\omega \subset \Omega$. We want to develop a finite element method that provides an approximation for the solution $u$ on a relatively coarse mesh on $\Omega$, certainly too coarse to resolve properly the highly oscillating features inside $\omega$, and then corrects in an iterative manner the numerical solution using another much finer local mesh on $\omega$. We introduce thus a coarse mesh $\mathcal{T}_{H}$ on $\Omega$ with a corresponding finite element space $V_{H}$ and a fine mesh $\mathcal{T}_{h}$ on $\omega$, from which is built a corresponding finite element space $V_{h}$. Let us suppose for the sake of simplicity that these spaces are based on $\mathbb{P}_{k}$-continuous Lagrange finite elements, with $k \geq 1$ (but this is not a restrictive assumption). We further denote $V_{H}^{0} \subset V_{H}$ and $V_{h}^{0} \subset V_{h}$ the subspaces of functions that vanish on $\partial \Omega$ and $\partial \omega$ respectively. We introduce also the finite element space $M_{h}$ on $\partial \omega$ as the discrete trace space of $V_{h}$ :

$$
M_{h}=\left\{\mu_{h} \in C^{0}(\partial \omega):\left.\mu_{h}\right|_{E} \in \mathbb{P}_{k}(E) \text { on every boundary edge (face) } E \text { of the mesh } \mathcal{T}_{h}\right\}
$$

A multiscale semi-discretization in space of problem (1) can be written as:

Find $u_{H}:[0, T] \rightarrow V_{H}^{0}, u_{h}:[0, T] \rightarrow V_{h}$, and $\lambda_{h}:[0, T] \rightarrow M_{h}$ that satisfy at all time $t \in(0, T):$ 


$$
\begin{aligned}
\int_{\Omega} \frac{\partial u_{H}}{\partial t} v_{H}+\int_{\Omega} \tilde{\mathbf{K}} \nabla u_{H} \cdot \nabla v_{H} & =\int_{\Omega \backslash \bar{\omega}} f v_{H}-\int_{\partial \omega} \lambda_{h} v_{H} \\
& +\int_{\omega} \frac{\partial u_{H}}{\partial t} v_{H}+\int_{\omega} \tilde{\mathbf{K}} \nabla u_{H} \cdot \nabla v_{H}, \forall v_{H} \in V_{H}^{0}, \\
\int_{\omega} \frac{\partial u_{h}}{\partial t} v_{h}+\int_{\omega} \mathbf{K} \nabla u_{h} \cdot \nabla v_{h}-\int_{\partial \omega} \lambda_{h} v_{h} & =\int_{\omega} f v_{h}, \quad \forall v_{h} \in V_{h}, \\
\int_{\partial \omega} u_{h} \mu_{h} & =\int_{\partial \omega} u_{H} \mu_{h}, \quad \forall \mu_{h} \in M_{h}, \\
u_{H}(\cdot, t=0)=u_{H}^{0}, & u_{h}(\cdot, t=0)=u_{h}^{0},
\end{aligned}
$$

where $u_{H}^{0}$ and $u_{h}^{0}$ are some discretizations of the initial condition $u_{0}$ (for instance the Lagrange interpolant or the $L^{2}$-projection), and where $\tilde{\mathbf{K}}$ is some smooth extension of $\mathbf{K}$ inside $\omega$, i.e. $\tilde{\mathbf{K}}=\mathbf{K}$ on $\Omega \backslash \bar{\omega}$ and $\tilde{\mathbf{K}}$ smoother than $\mathbf{K}$ on $\omega$. We note that $u_{H}$ is typically non unique, however its restriction on $\Omega \backslash \bar{\omega}$ is unique and provides here an approximation to the exact solution $u$. Inside $\omega$, the exact solution is approximated by $u_{h}$ while $u_{H}$ is purely fictitious there. Finally, $\lambda_{h}$ approximates the normal derivative of $u$ on $\partial \omega$.

\section{A Parareal time-discretization for multi-model numerical zoom}

We are going now to discretize $(3)-(6)$ in time. We imagine that some relatively big time-step $\Delta t$ is sufficient to discretize the coarse part of the solution $u_{H}$ so that, given $u_{H}^{0} \in V_{H}$, we will search for $u_{H}^{n} \in V_{H}(n=1,2, \ldots, N)$ which are approximations to $u_{H}\left(t_{n}\right)$ at $t=t_{n}=n \Delta t(T=N \Delta t)$. On the contrary some finer discretization in time may be needed for the fine part of the solution $u_{h}$ and we leave the derivatives in time in the corresponding equations. Thus, with the implicit Euler scheme we have the following problem for each time-slab $\left(t_{n-1}, t_{n}\right), n=1,2, \ldots, N$ :

Find $u_{H}^{n} \in V_{H}^{0}, u_{h}:\left[t_{n-1}, t_{n}\right] \rightarrow V_{h}$, and $\lambda_{h}:\left[t_{n-1}, t_{n}\right] \rightarrow M_{h}$ such that

$$
\begin{aligned}
\int_{\Omega} \frac{u_{H}^{n}-u_{H}^{n-1}}{\Delta t} v_{H}+\int_{\Omega} \tilde{\mathbf{K}} \nabla u_{H}^{n} \cdot \nabla v_{H} & =\int_{\Omega \backslash \bar{\omega}} f\left(t_{n}\right) v_{H}-\int_{\partial \omega} \lambda_{h}\left(t_{n}\right) v_{H} \\
& +\int_{\omega} \frac{u_{H}^{n}-u_{H}^{n-1}}{\Delta t} v_{H}+\int_{\omega} \tilde{\mathbf{K}} \nabla u_{H}^{n} \cdot \nabla v_{H}, \forall v_{H} \in V_{H}^{0}, \\
\int_{\omega} \frac{\partial u_{h}}{\partial t} v_{h}+\int_{\omega} \mathbf{K} \nabla u_{h} \cdot \nabla v_{h}-\int_{\partial \omega} \lambda_{h} v_{h} & =\int_{\omega} f v_{h}, \quad \forall v_{h} \in V_{h} \text { for all } t \in\left(t_{n-1}, t_{n}\right), \\
\int_{\partial \omega} u_{h} \mu_{h} & =\int_{\partial \omega} u_{H}^{n} \mu_{h}, \quad \forall \mu_{h} \in M_{h} \text { for all } t \in\left(t_{n-1}, t_{n}\right), \\
u_{h}\left(\cdot, t=t_{n-1}\right) & =U_{h}^{n-1} .
\end{aligned}
$$

In equation (10), the quantity $U_{h}^{n-1} \in V_{h}$ is a known initial condition. For a sequential time-integration method, it would be given by $U_{h}^{n-1}=u_{h}\left(t_{n-1}^{-}\right), n \geq 1$, and $U_{h}^{0}=u_{h}^{0}$.

Remark 1 Note that the (weak) boundary condition $u_{h}(t)=u_{H}^{n}, t \in\left(t_{n-1}, t_{n}\right)$, is rather arbitrary in the equation (9). We could use $u_{H}^{n-1}$ instead of $u_{H}^{n}$, for example, without loosing the accuracy a priori, or linear interpolation instead of a piecewise constant interpolation in time.

We propose the following iterative algorithm to compute the initial conditions $U_{h}^{n}, n=1,2, \ldots, N-1$ together with the coarse components $u_{H}^{n}$. We assume that, at each stage $k$ of the algorithm $(k \geq 0)$, we have already some approximations $\left(u_{H}^{k, n}\right)_{n=0, \ldots, N} \subset V_{H},\left(U_{h}^{k, n}\right)_{n=0, \ldots, N-1} \subset V_{h},\left(\Lambda_{h}^{k, n}\right)_{n=1, \ldots, N} \subset M_{h}$ that are compatible in the sense

$$
\left.U_{h}^{k, n}\right|_{\partial \omega}=P_{h, \partial \omega}\left(u_{H}^{k, n}\right), \quad n=0,1,2, \ldots, N-1
$$


where $P_{h, \partial \omega}$ is the $L^{2}(\partial \omega)$-projector onto $M_{h}$. We proceed as follows to find $\left(u_{H}^{k+1, n}\right)_{n=0, \ldots, N},\left(U_{h}^{k+1, n}\right)_{n=0, \ldots, N-1}$ and $\left(\Lambda_{h}^{k+1, n}\right)_{n=1, \ldots, N}$ :

\section{Parareal numerical zoom algorithm}

\section{Parallel fine computations on the patch}

Compute $u_{h}^{k+1, n}:\left[t_{n}, t_{n+1}\right] \rightarrow V_{h}$ and $\lambda_{h}^{k+1, n}:\left[t_{n}, t_{n+1}\right] \rightarrow M_{h}$ by solving (8)-(10):

$$
\begin{aligned}
\int_{\omega} \frac{\partial u_{h}^{k+1, n}}{\partial t} v_{h}+\int_{\omega} \mathbf{K} \nabla u_{h}^{k+1, n} \cdot \nabla v_{h}-\int_{\partial \omega} \lambda_{h}^{k+1, n} v_{h} & =\int_{\omega} f v_{h}, \quad \forall v_{h} \in V_{h} \text { for all } t \in\left(t_{n}, t_{n+1}\right), \\
\int_{\partial \omega} u_{h}^{k+1, n} \mu_{h} & =\int_{\partial \omega} u_{H}^{k, n+1} \mu_{h}, \quad \forall \mu_{h} \in M_{h} \text { for all } t \in\left(t_{n}, t_{n+1}\right), \\
\left.u_{h}^{k+1, n}\right|_{t=t_{n}} & =U_{h}^{k, n},
\end{aligned}
$$

for all $n=0,1,2, \ldots, N-1$.

\section{Sequential coarse computations and jump propagation}

Set, for $n=1,2, \ldots, N-1, S_{h}^{k, n}:=\left.u_{h}^{k+1, n-1}\right|_{t=t_{n}}-U_{h}^{k, n} \in V_{h}$, and $S_{h}^{k, 0}=0$.

(a) Compute the coarse solution $u_{H}^{k+1, n} \in V_{H}$ for $n=1,2, \ldots, N$ :

$$
\begin{aligned}
\int_{\Omega} \frac{u_{H}^{k+1, n}-u_{H}^{k+1, n-1}}{\Delta t} v_{H} & +\int_{\Omega} \tilde{\mathbf{K}} \nabla u_{H}^{k+1, n} \cdot \nabla v_{H}=\int_{\Omega \backslash \bar{\omega}} f\left(t_{n}\right) v_{H}-\int_{\partial \omega} \Lambda_{h}^{k, n} v_{H} \\
& +\int_{\omega} \frac{u_{H}^{k, n}-u_{H}^{k, n-1}}{\Delta t} v_{H}+\int_{\omega} \tilde{\mathbf{K}} \nabla u_{H}^{k, n} \cdot \nabla v_{H}, \forall v_{H} \in V_{H}^{0} .
\end{aligned}
$$

(b) Compute the corrections $\Delta U_{h}^{k+1, n} \in V_{h}$ and $\Delta \Lambda_{h}^{k+1, n} \in M_{h}$ for $n=1,2, \ldots, N$ :

$$
\begin{array}{r}
\int_{\omega} \frac{\Delta U_{h}^{k+1, n}-\Delta U_{h}^{k+1, n-1}}{\Delta t} v_{h}+\int_{\omega} \mathbf{K} \nabla\left(\Delta U_{h}^{k+1, n}\right) \cdot \nabla v_{h}-\int_{\partial \omega} \Delta \Lambda_{h}^{k+1, n} v_{h}=\frac{1}{\Delta t} \int_{\omega} S_{h}^{k, n-1} v_{h}, \\
\forall v_{h} \in V_{h}, \quad(13) \\
\int_{\partial \omega} \Delta U_{h}^{k+1, n} \mu_{h}=\int_{\partial \omega}\left(u_{H}^{k+1, n}-u_{H}^{k, n}\right) \mu_{h}, \quad \forall \mu_{h} \in M_{h} .
\end{array}
$$

\section{Correction of the initial values}

Update for $n=1,2, \ldots, N$ :

$$
\begin{aligned}
U_{h}^{k+1, n} & =\left.u_{h}^{k+1, n-1}\right|_{t=t_{n}}+\Delta U_{h}^{k+1, n}, \\
\Lambda_{h}^{k+1, n} & =\left.\lambda_{h}^{k+1, n-1}\right|_{t=t_{n}}+\Delta \Lambda_{h}^{k+1, n} .
\end{aligned}
$$

Concerning the initialization of the algorithm, at $k=0$, we can solve (1) directly on the coarsest grid (time-step $\Delta t$ and finite element space $V_{H}$ ), and with $\tilde{\mathbf{K}}$ as a diffusion matrix, so as to obtain $u_{H}^{0, n}$. Then $U_{h}^{0, n}$ and $\Lambda_{h}^{0, n}$ are obtained by solving sequentially the fine problem on the patch with the coarsest time-step $\Delta t$ (but still with the finite element space $V_{h}$ and the multiscale diffusion matrix $\mathbf{K}$ ), so that property (11) is ensured initially, while the computational cost of the initialization remains of the same order than the cost of the sequential steps 2 (a) and (b) in our algorithm.

Upon convergence we have hopefully $S_{h}^{\infty, n}=0$ so that $\left.u_{h}^{\infty, n-1}\right|_{t=t_{n}}=U_{h}^{\infty, n}$ and putting all the $u_{h}^{\infty, n}$ together we obtain the exact solution to (7)-(10). In practice, we can stop the algorithm after a few correction iterations, or when the norm of the jumps $\left(S_{h}^{k, n}\right)$ is below a given threshold. 
Note finally that the proposed algorithm preserves the relationship (11):

Proposition 3.1 The discrete solution $\left(U_{h}^{k, n}, u_{H}^{k, n}\right)$ satisfies for all $k \geq 0$ and $n=0,1,2, \ldots, N-1$ :

$$
\left.U_{h}^{k, n}\right|_{\partial \omega}=P_{h, \partial \omega}\left(u_{H}^{k, n}\right) .
$$

Proof. Assume that (11) holds for a given $k \geq 0$, then (16) holds also due to both relationships (14) and (15).

Remark 2 The computation of the corrections $\Delta U_{h}^{k+1, n}$ and $\Delta \Lambda_{h}^{k+1, n}$ effectuated in equation (13) is motivated by the following: if we put together all the contributions $u_{h}^{k+1, n}$ into a single function $u_{h}^{k+1}$ : $[0, T] \rightarrow V_{h}$, we observe that it satisfies (4) with the extra term $-\sum_{n=1}^{N-1} \delta\left(t-t_{n}\right) \int_{\omega} S_{h}^{k, n} v_{h}$, where $\delta\left(\cdot-t_{n}\right)$ is the Dirac mass at $t_{n}$. Therefore we proceeded exactly as in the first version of the Parareal method described in [10] for an ordinary differential equation.

Remark 3 Let us consider the case where the equations for $u_{h}^{k+1, n}$ are discretized by implicit Euler on the same coarse mesh $\Delta t$ as those for $u_{H}$, i.e. $\left.u_{h}^{k+1, n-1}\right|_{t=t_{n}}$ are obtained from $U_{h}^{k, n-1}$ via

$$
\begin{aligned}
\int_{\omega} \frac{\left.u_{h}^{k+1, n-1}\right|_{t=t_{n}}-U_{h}^{k, n-1}}{\Delta t} v_{h}+\int_{\omega} \mathbf{K}\left(\left.\nabla u_{h}^{k+1, n-1}\right|_{t=t_{n}}\right) \cdot \nabla v_{h}-\int_{\partial \omega} \hat{\lambda}_{h}^{k+1, n} v_{h} & =\int_{\omega} f v_{h}, \quad \forall v_{h} \in V_{h}, \\
\int_{\partial \omega}\left(\left.u_{h}^{k+1, n-1}\right|_{t=t_{n}}\right) \mu_{h} & =\int_{\partial \omega} u_{H}^{k, n} \mu_{h}, \quad \forall \mu_{h} \in M_{h} .
\end{aligned}
$$

Here $\hat{\lambda}_{h}^{k+1, n}$ is in fact an approximation to $\lambda_{h}^{k+1, n-1}\left(t_{n}\right)$. Combining the above equation with (12), (13), (14) and (15) we observe that the algorithm can be rewritten in terms of only $u_{H}^{k, n}, U_{h}^{k, n}$ and $\Lambda_{h}^{k, n}$ :

$$
\begin{aligned}
& \int_{\Omega} \frac{u_{H}^{k+1, n}-u_{H}^{k+1, n-1}}{\Delta t} v_{H}+\int_{\Omega} \tilde{\mathbf{K}} \nabla u_{H}^{k+1, n} \cdot \nabla v_{H} \\
&=\int_{\Omega \backslash \bar{\omega}} f\left(t_{n}\right) v_{H}-\int_{\partial \omega} \Lambda_{h}^{k, n} v_{H} \\
&+\int_{\omega} \frac{u_{H}^{k, n}-u_{H}^{k, n-1}}{\Delta t} v_{H}+\int_{\omega} \tilde{\mathbf{K}} \nabla u_{H}^{k, n} \cdot \nabla v_{H}, \forall v_{H} \in V_{H}^{0}, \\
& \int_{\omega} \frac{U_{h}^{k+1, n}-U_{h}^{k+1, n-1}}{\Delta t} v_{h}+\int_{\omega} \mathbf{K} \nabla U_{h}^{k+1, n} \cdot \nabla v_{h}-\int_{\partial \omega} \Lambda_{h}^{k+1, n} v_{h}=\int_{\omega} f\left(t_{n}\right) v_{h}, \quad \forall v_{h} \in V_{h}, \\
& \int_{\partial \omega} u_{h}^{k+1, n} \mu_{h}=\int_{\partial \omega} u_{H}^{k+1, n} \mu_{h}, \quad \forall \mu_{h} \in M_{h} .
\end{aligned}
$$

\section{A numerical experiment}

We solve problem (1) with our algorithm, and with $d=2, \Omega=\left[-\frac{1}{4} ; \frac{3}{4}\right]^{2}, \omega=\left[0 ; \frac{1}{2}\right]^{2}, T=0.1$. The expression of the diffusion matrix is:

$$
\mathbf{K}(x, y, t)=\left(K(x, y) \mathbb{1}_{\hat{\omega}}(x, y)+1\right) \mathbf{I},
$$

where $K(x, y)=100 H(\sin 20 \pi x) H(\sin 20 \pi y), H$ is the Heaviside function, $\hat{\omega}=[2 / 10 ; 3 / 10]^{2} \subset \omega$ and $\mathbf{I}$ is the identity matrix in two dimensions. The source term is $f(x, y, t)=\mathbb{1}_{\hat{\omega}}(x, y)(10+20 \sin (226 \pi t))$. The initial condition is $u^{0}=0$. Note that the diffusion matrix, resp. the source term, is highly oscillating in space, resp. in time, only on the patch $\omega$. Finite element discretization is carried out with piecewisecontinuous $\mathbb{P}_{1}$ Lagrange finite elements, and with structured meshes of sizes $H=4 h=\frac{1}{8}$. For timediscretization, the coarse time-step is $\Delta t=0.005(N=20)$, and the fine problem (8)-(9) is discretized also with implicit Euler, and a finer time-step $\delta t=\frac{\Delta t}{10}$. Moreover, for the coarse solver, we take $\tilde{\mathbf{K}}(x, y, t)=\mathbf{I}$. Numerical experiments are carried out with FreeFEM++ [7].

We compare the solution to a reference that is obtained through direct time-discretization of Problem (3)-(6), using the finest time-step $\delta t$ (5 iterations between coarse and fine solvers are carried out at each 
time-step). The results are depicted Figure 1, where the solution $u_{c}\left(t^{n}\right)=u_{h}^{k, n}\left(x=\frac{1}{4}, y=\frac{1}{4}\right)$ at the center of the domain $\Omega$ is displayed, as a function of time, for the reference and various Parareal iterations $k$. We also display the coarse solution (left panel, 'Coarse'), which ignores completely the (spatial and temporal) multiscale effects (recall that $\tilde{\mathbf{K}}$ is smooth, and $\hat{\omega} \subset \omega)$. The initial solution $(k=0)$ is far away from the reference since it is computed with the coarsest time-step $\Delta t$. After the first Parareal correction iteration $k=1$ (right panel), the fine temporal multiscale effects are recovered, but there are still important jumps at the interface between the piecewise fine solutions. After the second Parareal correction $k=2$, we get very close to the sequential reference and there is no visible evolution of the solution when $k$ is increased. After $k=9$ iterations, the algorithm is stopped.
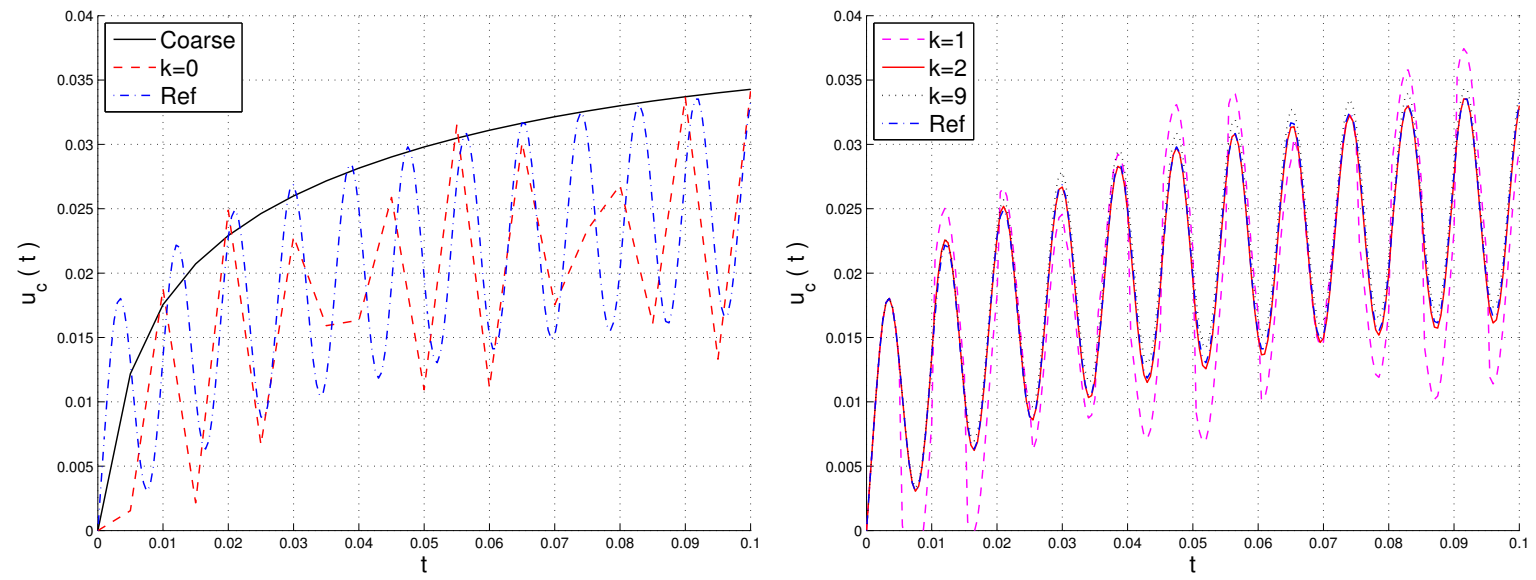

Figure 1. Evolution of the solution $u_{c}$ at the center of the domain $\Omega$, for various Parareal iterations $k$ and for reference (sequential) solution.

Finally, the table below presents the evolution of the jumps $\left(S_{h}^{k, n}\right)$ at each correction iteration $k$, which appear to converge to 0 .

\begin{tabular}{c|cccccccccc}
$\mathrm{k}$ & 0 & 1 & 2 & 3 & 4 & 5 & 6 & 7 & 8 & 9 \\
\hline $\log _{10}\left\|S_{h}^{k}\right\|_{L^{2}(0, T ; \omega)}$ & -4.91 & -4.36 & -5.75 & -6.53 & -7.16 & -7.27 & -7.78 & -7.94 & -8.34 & -8.57
\end{tabular}

\section{Acknowledgements}

The first author thanks Florian Lemarié for discussion and comments.

\section{References}

[1] J.-B. Apoung Kamga and O. Pironneau. Numerical zoom for multiscale problems with an application to nuclear waste disposal. J. Comput. Phys., 224(1):403-413, 2007.

[2] M. Astorino, F. Chouly, and A. Quarteroni. Multiscale coupling of finite element and lattice Boltzmann methods for time dependent problems. Submitted. http://hal.archives-ouvertes.fr/hal-00746942.

[3] G. Bal and Y. Maday. A "parareal" time discretization for non-linear PDE's with application to the pricing of an American put. In Vol. 23 of Lecture Notes in Computational Science and Engineering, Springer Verlag, Berlin, pages 189-202, 2002. 
[4] C. Farhat and M. Chandesris. Time-decomposed parallel time-integrators: theory and feasibility studies for fluid, structure, and fluid-structure applications. Int. J. Num. Meth. Engrg., 58:1397-1434, 2003.

[5] M.J. Gander and S. Vandewalle. Analysis of the parareal time-parallel time-integration method. SIAM J. Sci. Comp., 29:556-578, 2007.

[6] R. Glowinski, J. He, A. Lozinski, J. Rappaz, and J. Wagner. Finite element approximation of multi-scale elliptic problems using patches of elements. Numer. Math., 101(4):663-687, 2005.

[7] F. Hecht, O. Pironneau, J. Morice, A. Le Hyaric, and K. Ohtsuka. Freefem++ documentation, third edition, version 3.19-1, 2012. Webpage: http://www.freefem.org/ff++.

[8] P. Laborde and A. Lozinski. Numerical zoom for multi-scale and multi-model problems. In Preparation.

[9] F. Legoll, T. Lelièvre, and G. Samaey. A micro-macro parareal algorithm: application to singularly perturbed ordinary differential equations. SIAM J. Sci. Comput., 35(4):A1951-A1986, 2013.

[10] J.-L Lions, Y. Maday, and G. Turinici. Résolution d'EDP par un schéma en temps "pararéel". C. R. Acad. Sci. Paris, Ser. I. Math., 332:661-668, 2001.

[11] A. Lozinski. Méthodes numériques et modélisation pour certains problèmes multi-échelles. Habilitation à Diriger des Recherches, Université Paul Sabatier, Toulouse 3, 2010.

[12] F.D.J.L. Steger and J. Benek. A chimera grid scheme. In Advances in Grid Generation, K.N. Chis and U. Ghia ed., ASME FED-Vol. 5, 1983. 